\title{
Afrontamiento al estrés y modelo psicobiológico de la personalidad en estudiantes universitarios
}

\author{
Coping and psychobiological model of \\ personality in college students
}

\author{
Juan C. Espinosa* \\ Universidad Santo Tomás, \\ Bogotá, Colombia \\ Francoise V. Contreras \\ Universidad del Rosario, \\ Bogotá, Colombia \\ Gustavo A. Esguerra \\ Universidad Santo Tomás, \\ Bogotá, Colombia
}

Recibido: 23 de noviembre de 2008

Revisado: 4 de diciembre de 2008

Aceptado: 10 de enero de 2009

\section{Resumen}

El propósito de este estudio fue observar la relación entre las dimensiones de personalidad propuestas por el Modelo de Temperamento/Carácter y las dimensiones del Afrontamiento al Estrés en una muestra aleatoria estratificada de 150 de estudiantes universitarios. Los resultados indican que los participantes tienden a usar estrategias activas para afrontar el estrés; asimismo, se confirman los supuestos biológicos y culturales del Modelo de Personalidad y se evidencia que el afrontamiento al estrés guarda estrecha relación con las características de personalidad, en especial con dos dimensiones del Carácter (Autodirectividad y Auto-trascendencia) y poca relación con las dimensiones del temperamento en especial con evitación del daño y persistencia. Se discuten las implicaciones de estos resultados.

Palabras clave: afrontamiento al estrés, personalidad, modelo de temperamento y carácter.

\footnotetext{
Correspondencia: Juan Carlos Espinosa, Facultad de Psicología, Universidad Santo Tomás. Dirección: Cra. 9 No. 51-11, Bogotá, Colombia. Grupo de investigación Psicología, Salud y Calidad de vida. Correo electrónico: juanespinosa@usantotomas.edu.co. Francoise Contreras Torres: Grupo de investigación GIPE. Centro de Estudios Empresariales para la Perdurabilidad (CEEP). Correo electrónico: francoise.contreras@urosario.edu.co. Gustavo Adolfo Esguerra Pérez: Grupo de investigación Psicología, Salud y Calidad de vida. Correo electrónico: gustavoesguerra@usantotomas.edu.co.
} 


\section{Abstract}

The purpose of this study was to observe the relationship between personality dimensions suggested for Temperament/Character Model and Coping in 150 undergraduate students in a randomized stratified sample. Results indicate that participants tended to use active strategies for coping. Assumptions about biological and cultural aspects of the Personality Model are confirmed and there is evidence that coping is closely related to personality traits, especially to Character dimensions (Self directiveness and Self transcendence), and to Temperament dimensions especially to avoidance. The implications of these results are discussed.

Key words: coping, personality, temperament and character model.

\section{Introducción}

Los estilos de afrontamiento se definen como "aquellos esfuerzos cognitivos y conductuales constantemente cambiantes que se desarrollan para manejar las demandas específicas externas y/o internas que son evaluadas como excedentes o desbordantes de los recursos del individuo" (Lazarus \& Folkman, 1986, p. 164). Dichos estilos suponen el esfuerzo del individuo para hacer frente a los estímulos estresantes y tolerar sus efectos de la manera menos perjudicial (Matheny, Aycock, Pugh, Curlette \& Silva-Canella, 1986), y constituyen la forma habitual en la que el individuo se enfrenta a los eventos percibidos como estresantes (Caballo, 1998), lo cual supone el reconocimiento y uso de recursos psicológicos de los que se disponga en un momento dado.

El afrontamiento implica una serie de procesos cognitivos y conductuales estrechamente relacionados; por una parte, dependen de la evaluación que realiza el sujeto sobre la situación y, por otra, del uso de estrategias conductuales utilizadas para aliviar la carga que supone el estresor sobre el bienestar psicológico (Mok \& Tam, 2001 Richardson \& Poole, 2001). Hay algunos estilos que pueden ser funcionales a corto plazo en cuanto logran el objetivo de disminuir el impacto del estresor, pero a largo plazo pueden comprometer de manera considerable la calidad de vida, la salud mental y el bienestar psicológico general de las personas (Wahl, Hanestad, Wiklund \& Poum, 1999).
De acuerdo con la literatura, los estilos de afrontamiento activos, aquéllos que implican una confrontación adecuada de las propias emociones, se encuentran relacionados con mayor bienestar psicológico y estados afectivos positivos (Carrobles, Remor \& Rodríguez-Alzamora, 2003). Entre ellos están la reinterpretación positiva, en la que el individuo se centra en los aspectos positivos de la situación (Gunzerath, Connelly, Albert \& Knebel, 2001), y el afrontamiento centrado en la solución del problemas que se han relacionado con mayor salud mental (Contreras, Esguerra, Espinosa \& Gómez, 2007). Asimismo, el afrontamiento que involucra estados optimistas en torno a la situación, favorece la salud mental y la percepción de bienestar y calidad de vida de los individuos (Van der Zaag-Loonen, Grootenhuis, Last \& Derkx, 2004; Wu, Lee, Baig \& Wichaikhum, 2001); estos aspectos son relevantes en la medida en que proporcionan conocimiento sobre características benignas y positivas para las personas (Contreras \& Esguerra, 2006).

Por otra parte, los estilos pasivos implican una tendencia a perder el control sobre la situación, se caracterizan por estados de depresión y afecto negativo (Brown, Nicassio \& Woolston, 1989). Entre ellos está la autofocalización negativa, caracterizada por indefensión, culpa, sentimientos de incapacidad para afrontar la situación y creencia de que las cosas suelen salir mal, este estilo de afrontamiento afecta de manera considerable varias dimensiones de la calidad de vida percibida (Contreras et al., 2007). Las estrategias cen- 
tradas en la emoción, que pretenden evitar los problemas, y que se acompañan de una fuerte tendencia a catastrofizar, se han relacionado con síntomas depresivos, menor percepción de apoyo social, ansiedad, percepción de malestar psicológico y detrimento en la calidad de vida percibida (Arraras, Wrigth, Jusue, Tejedor \& Calvo, 2002; Griswold, Evans, Spielman \& Fishman, 2005).

Dado que los estilos de afrontamiento promueven los recursos del paciente y pueden afectar el bienestar psicológico, es de especial interés conocer qué variables psicológicas se relacionan con los estilos adecuados e inadecuados de afrontamiento. Conocer estas variables, puede ser útil para el desarrollo de intervenciones que potencien los recursos psicológicos de los jóvenes y favorezcan su bienestar percibido, lo que en alguna medida puede tener efecto sobre aquellas problemáticas que en cierta forma involucran estilos de afrontamiento no adaptativos frente a las múltiples demandas a las que se ve expuesta esta población, en razón de las variables psicosociales que la caracterizan.

Dentro de las variables que pueden afectar los estilos de afrontamiento se encuentra la personalidad. Se ha evidenciado que el afrontamiento al estrés muestra diferencias individuales que pueden ser mediadas por características de personalidad (Gunzerath et al., 2001); en este estudio la personalidad se estimó de acuerdo con el Modelo Psicobiológico del Temperamento y el Carácter planteado por Cloninger, Svrakic y Przybeck (1993), que se ubica dentro de la corriente psicobiológica iniciada por Eysenck en la década del cuarenta y continuada posteriormente por Gray y Zuckerman en la década del ochenta (Espinosa \& Herrera, 2003; Díaz \& Pizarro, 2000). Los desarrollos conceptuales y metodológicos en esta corriente han identificado siete dimensiones que permiten una razonable descripción y explicación de la personalidad (Cloninger, Przybeck, Svrakic, \& Wetzel, 1994).

Este modelo supone dos componentes estructurales de la personalidad: temperamento y carácter. El primero alude al componente biológico, y plantea que existen sistemas cerebrales independientes para activación, mantenimiento e inhibición de la conducta en respuesta a una clase específica de estímulos. Por su parte, el carácter hace referencia al componente sociocultural de la personalidad, el cual contempla que los conceptos sobre sí mismo, los demás y el universo, modulan el significado de lo experimentado y, en consecuencia, las reacciones emocionales de las personas (Cloninger et al., 1994).

El temperamento, heredable en cierto grado (Health, Cloninger \& Martin, 1994), comprende predisposiciones preconceptuales en la memoria y la formación de hábitos (Cloninger, 1994), e incluye cuatro dimensiones: la búsqueda de novedad, evitación al daño, dependencia de refuerzos y persistencia. El carácter corresponde a la formación de conceptos que maduran en la adultez y que influyen en la manera en que la persona se relaciona consigo misma y con el entorno; incluye tres dimensiones: la autodirectividad, la cooperatividad y la autotrascendencia (Cloninger et al., 1993).

El propósito de este estudio es describir los estilos de afrontamiento al estrés en un grupo de estudiantes universitarios y observar si éstos se relacionan con las dimensiones del Modelo del Temperamento y Carácter de Cloninger et al., (1994). Asimismo, se pretende buscar evidencia que ayude a la comprensión de las diferencias individuales en la utilización de las diferentes estrategias de afrontamiento al estrés.

\section{Método}

\section{Tipo de estudio}

\section{Descriptivo correlacional.}

\section{Participantes}

La muestra estuvo conformada por 150 estudiantes de psicología de una universidad privada de Bogotá, D.C., seleccionados con muestreo aleatorio estratificado por semestre; los participantes, tenían una edad promedio de 21,5 (d.t. 3,4) años, y la mayoría eran mujeres (82\%). Los estudiantes 
decidieron participar voluntariamente en la investigación, previo consentimiento informado.

\section{Instrumentos}

Cuestionario de Afrontamiento al estrés (CAE): es una escala de 42 ítems desarrollada y validada por Sandín y Chorot (2003). El instrumento fue diseñado para evaluar siete estilos básicos de afrontamiento: 1) focalizado en la solución del problema (FSP), 2) autofocalización negativa (AFN), 3) reevaluación positiva (REP), 4) expresión emocional abierta (EEA), 5) evitación (EVT), 6) búsqueda de apoyo social (BAS), y 7) religión (RLG). El instrumento ha mostrado adecuadas propiedades psicométricas, y obtuvo un coeficiente promedio de fiabilidad de Cronbach de 0,79 para las siete subescalas (Sandín \& Chorot, 2003). Los estudios realizados con el CAE en población colombiana demuestran alta confiabilidad y validez en sus dimensiones (Cano et al., 2006; Contreras et al., 2007).

Inventario de Personalidad [TCl-125]: se utilizó la versión reducida (125 ítems) del Inventario de Personalidad TCI-9 de Cloninger et al., (1994), adaptado para población colombiana por Díaz \& Chaparro (2000). El TCl estima cuatro dimensiones del temperamento y tres del carácter. Las características de las cuatro dimensiones del temperamento y las tres del carácter son descritas por Cloniger et al. (1994) de la siguiente manera:

- Evitación al daño (HA, por su nombre en inglés Harm Avoidance). Los individuos que tienen altas puntuaciones en esta escala tienden a ser precavidos, cuidadosos, miedosos, tensos, aprensivos, nerviosos, tímidos, dudosos, inseguros, pasivos, negativos, malhumorados o pesimistas, incluso, en situaciones que no representen preocupación para otros. Sus niveles de energía tienden a ser bajos y se sienten fatigados o cansados con facilidad. Los que puntúan bajo tienden a ser despreocupados, relajados, valientes, optimistas, atrevidos y calmados, incluso en situaciones en las que la mayoría de las personas se preocuparía. Sus niveles de energía tienden a ser altos y son considerados por los demás como vigorosos y dinámicos.

- Búsqueda de novedad (NS, Novelty Seeking). Los que puntúan alto tienden a caracterizarse por ser excitables, exploradores, curiosos, entusiastas, exuberantes, se aburren fácilmente, son impulsivos y desordenados. Por el contrario, los que puntúan bajo son reflexivos, reservados, estoicos, poco entusiastas, frugales, toleran la monotonía, son sistemáticos y organizados.

- Dependencia del refuerzo (RD, Reward Dependence). Las personas que tienen puntuaciones altas en este factor tienden a ser sentimentales, amorosos y cálidos, dedicados, dependientes y sociables. Por su parte, quienes puntúan bajo tienden a ser prácticos, racionales, fríos y socialmente insensibles.

- Persistencia (PS, Persistence). Las personas que puntúan alto en persistencia tienden a ser trabajadoras, persistentes, esforzadas, y estables a pesar de la frustración y la fatiga. Intensifican sus esfuerzos anticipando recompensas, se presentan voluntariamente para realizar cosas y son vehementes para comenzar con los deberes.

- Autodirectividad (SD, Self-directedness). Las personas que puntúan alto en esta dimensión asumen las consecuencias de sus conductas sin culpar a los demás, tienen metas claras y actúan de forma consecuente para alcanzarlas, se sienten con los recursos y hábitos necesarios para ser efectivas en sus planes y tienen un alto concepto de sí mismas. En general, son descritos como personas maduras. Por el contrario, quienes puntúan bajo son personas que no encuentran un propósito definido para su vida, por lo cual no se plantean metas ni hábitos para cumplir con algún plan establecido, tienden a culpar a los demás por sus conductas inefectivas y el concepto propio suele ser muy bajo.

- Cooperatividad (C, Cooperativeness). Aquéllos que puntúan alto aceptan a las demás personas a pesar de ser muy distintas a ellos, tratan de ponerse en lugar de los otros en ciertas situaciones, no tratan de tomar ventaja, son compasivos en vez de intentar vengarse si alguien les ha hecho daño y son generosos 
y bondadosos. Las personas que sólo piensan en aprovecharse de los demás, en vengarse cuando los han ofendido, y que son egoístas e intolerantes con personas distintas a ellas obtienen puntajes bajos en esta dimensión.

- Autotrascendencia (ST, Self-Transcendence). El auto-olvido es una característica de ciertas personas para involucrarse tanto en lo que se hallan haciendo que olvidan por un momento el tiempo y el lugar en el que se encuentran, generalmente ocurre en momentos de creatividad. Se encuentran puntajes altos de autotrascendencia en personas que reportan este tipo de experiencia, así como preocupación por temas que atañen a todas las personas, como es el cuidado del medio ambiente y de especies en vías de extinción. Son personas que valoran y son muy sensibles al arte y a la naturaleza, conciben la idea de una fuerza espiritual que sostiene o guía sus vidas y creen en poderes extrasensoriales como la telepatía, o que pueden ocurrir milagros. En oposición, las personas que puntúan bajo son personas que se encuentran conscientes de sí en todo momento, no reportan olvidar el lugar ni el tiempo mientras realizan alguna actividad, son poco sensibles a las obras de arte o a la naturaleza, piensan únicamente en sus propios asuntos y no creen que exista una fuerza superior ni poderes extrasensoriales.

\section{Procedimiento}

Una vez obtenido el consentimiento informado por parte de los estudiantes, se procedió a la aplicación de los instrumentos de forma colectiva. A los participantes se les aclaró el manejo ético de la información, y que no recibirían remuneración económica por su participación; asimismo, se les recordó que podrían rehusarse a diligenciar los instrumentos si lo consideraban conveniente.

\section{Resultados}

En el orden descriptivo se observa un mayor uso de estilos de afrontamiento activos, centrados en el problema, reevaluación positiva y búsqueda de apoyo social, y un menor uso de aquellos estilos considerados pasivos y centrados en la emoción, esto es, expresión emocional abierta, autofocalización negativa y religiosidad (figura 1). La evitación, que pertenece también a esta última categoría, puntuó alto, aspecto que debe ser estudiado, en cuanto constituye un estilo no funcional ni adaptativo.

Los resultados obtenidos en el $\mathrm{TCl}$ por la muestra de estudiantes colombianos se comparó con otros datos obtenidos con estudiantes anglosajones (Cloninger et al., 1994). Se observa un patrón muy similar en Búsqueda de Novedad (NS) y Evitación al Daño (HA), persistencia (PS) y Auto-Trascendencia (ST); una leve diferencia en Dependencia del Refuerzo (RD); y fuertes diferencias en Autodirectividad (SD) y Cooperatividad (C), ambas, dimensiones del carácter que en cuanto tal involucran aspectos socioculturales (figura 2 ).

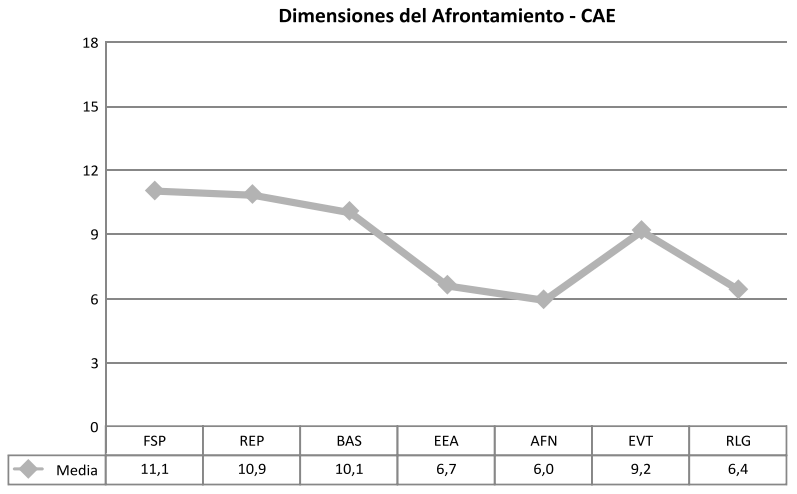

Figura 1. Dimensiones del afrontamiento al estrés utilizados por los estudiantes

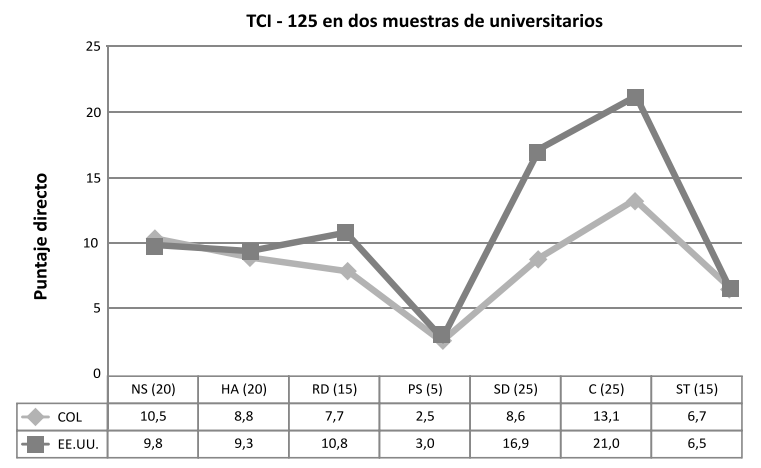

Figura 2. Puntuaciones obtenidas en el $\mathrm{TCl}$ por los participantes del estudio muestra colombiana) y los obtenidos por estudiantes anglosajones 
Por otra parte, al examinar las asociaciones entre las dimensiones del afrontamiento estimadas con el CAE y las dimensiones de la personalidad evaluadas con $\mathrm{TCl}-125$ se encontraron asociaciones estadísticamente significativas, sólo una de ellas de tipo negativo, la cual se presenta entre el afrontamiento Focalizado en la Solución de Problemas (FSP) y la Autodirectividad. Las otras asociaciones estimadas indican que la Reevalua- ción Positiva (REP) se asocia con Autotrascendencia; la Búsqueda de Apoyo Social (BAS) se correlaciona con Búsqueda de Novedad, Dependencia del Refuerzo y Autotrascendencia; la Expresión Emocional Abierta (EEA) se relaciona con Autodirectividad y Cooperatividad; la Autofocalización Negativa (AFN) se asocia con Autodirectividad; y tanto la Evitación (EVT) como la Religión (RLG) se correlacionan con Auto-trascendencia (tabla 1).

Tabla 1. Correlación entre las dimensiones de personalidad y los estilos de afrontamiento

\begin{tabular}{lccccccc}
\hline & \multicolumn{7}{c}{ Dimensiones del Afrontamiento al Estrés } \\
$\begin{array}{l}\text { Dimensiones del Tempera- } \\
\text { mento y el Carácter }\end{array}$ & FSP & REP & BAS & EEA & AFN & EVT & RLG \\
\hline Búsqueda de Novedad [NS] & - & - &, 318 & - & - & - & - \\
Evitación del daño [HA] & - & - & - & - & - & - & - \\
Depend. del Refuerzo [RD] & - & - &, 228 & - & - & - & - \\
Persistencia [ PS ] & - & - & - & - & - & - & - \\
Auto-directividad [SD] &,- 286 & - & - &, 269 &, 441 & - & - \\
Cooperatividad [ C ] & - & - & - &, 208 & - & - & - \\
Auto-trascendencia [ST] & - &, 229 &, 319 & - & - &, 193 &, 324 \\
\hline
\end{tabular}

Nota: todas las correlaciones son significativa al nivel 0,01 (bilateral), exceptuando C-EAA y ST-EVT que son significativas al nivel 0,05 (bilateral).

De acuerdo con estos resultados, algunas dimensiones de personalidad, principalmente las vinculadas al carácter, guardan relación con la forma habitual en que los individuos afrontan los eventos que perciben como estresantes, exceptuando la persistencia y evitación al daño (pertenecientes al temperamento), que no se relacionaron con ninguno de los estilos de afrontamiento estudiados.

\section{Discusión}

Afrontar las distintas demandas del ambiente, muchas de ellas altamente estresantes, implica que los jóvenes hagan uso de los recursos psicológicos de los que dispone para aliviar el impacto del estresor. El uso de determinados estilos de afrontamiento depende de la forma en que el individuo evalúa la situación, e implica un repertorio conductual en cierta medida estable, que hace parte de la forma habitual en que se suele responder a las demandas del ambiente, sea o no adaptativo (aunque sí funcional) para el individuo.

Los participantes de este estudio suelen afrontar los eventos estresantes de manera activa, lo cual constituye un recurso importante en esta población. Se encontró el uso frecuente de estrategias activas de afrontamiento (FSP, REP y BAS) en esta muestra, aspecto que resulta favorable tanto para el bienestar psicológico como para la calidad de vida, en general. No obstante, la evitación como afrontamiento, obtuvo una puntuación alta, aspecto que debe ser estudiado y observar su posible relación con comportamientos no deseados en jóvenes, tanto en el ámbito académico como personal.

Las dimensiones del Temperamento (NS, HA, RD y PS) presentan gran similitud con la población anglosajona, lo cual confirma el supuesto biológico 
de estas dimensiones. En cambio, las dimensiones del carácter (SD y C) presentan diferencias marcadas frente a las de EE.UU., lo cual refleja diferencias culturales que son más claras en el caso de SD y menos en C, probablemente debido a que la cultura anglosajona tiende a ser más independiente y menos gregaria que la cultura latina.

En el aspecto descriptivo, una parte que vale la pena resaltar es un mayor nivel en Búsqueda de Novedad, en relación con una baja puntuación en la dimensión persistencia, que parece asociarse con conductas no deseadas. De acuerdo con Gómez, Pérez, Portolés y Salom (2001) el aumento en la dimensión de Búsqueda de Novedad pondría de manifiesto un comportamiento propenso a desarrollar actividades que entrañan peligro, siguiendo el impulso sin observar reglas, unido a una baja persistencia reflejaría una menor capacidad de consecución y perseverancia, lo que podría explicar la alta de recaídas en personas con adicción a las drogas. Si se relaciona este aspecto con la tendencia a la evitación como estrategia de afrontamiento puede llegar a ser un factor de riesgo para el consumo de sustancias psicoactivas, tal como el alcohol, el tabaco, entre otras; por tal razón, es importante generar estrategias que conlleven el afrontamiento activo del estrés.

Con respecto a la relación entre estrategias de afrontamiento y personalidad, se encontró la mediación de dos dimensiones del temperamento y tres del carácter, con el uso de los distintos estilos de afrontamiento evaluados. Entre estos tenemos que la Búsqueda de Apoyo Social, considerada una dimensión activa de afrontamiento; se relacionó con Búsqueda de Novedad, Dependencia del Refuerzo y Auto-trascendencia. Estos resultados son comprensibles teóricamente, pues todas estas dimensiones implican una búsqueda activa de recursos y apoyo, ya sea social o espiritual.

Resultó de gran interés conceptual fue el hallazgo de que a mayor autodirectividad (característica del carácter), menor uso de estilos de afrontamiento centrados en la solución de problemas, y mayor uso de estilos pasivos y emotivos como la Expresión Emocional Abierta (EEA) y Autofocalización Negativa (AFN). La relevancia está en que si se toman aisladamente, la asociación negativa entre Autodirectividad (SD) y Focalización en la Solucion del Problema (FSP) es incoherente; pero si se tiene en cuenta, por un lado, que la principal estrategia de afrontamiento usada por la muestra es la FSP y que, a su vez, presentan una tendencia baja en SD y, por el otro, se tiene en cuenta el poco uso de estrategias pasivas tal como EEA y AFN, entonces estas asociaciones son teóricamente consistentes, pues la baja autodirectividad está asociada con el uso de estrategias pasivas y la correlación negativa entre SD y FSP se debe principalmente a la baja magnitud de atributo de esta dimensión del carácter en comparación con otras muestras estudiadas.

Otro aspecto de interés teórico y que aporta a la solidez del modelo, en especial a la inclusión de la dimensión espiritual en un modelo de personalidad, fueron las cuatro asociaciones que presentó la autotrascendencia, con los estilos de afrontamiento, reevaluación positiva, búsqueda de apoyo social, evitación y religiosidad, dos estilos de afrontamiento activos y dos estilos pasivos. Este resultado indica que la alta magnitud de autotrascedencia favorece, ya sea la reinterpretación o revaloración de las situaciones estresantes como algo favorable, que fortalece el carácter, o encamina a las personas a buscar apoyo en sus congéneres. No obstante, este apoyo también se puede esperar de figuras divinas $y$, por ende, estaría relacionada con la religiosidad y la evitación del problema. Por tal razón, esta dimensión de personalidad debe ser estudiada con mayor detenimiento, para identificar hasta qué punto favorece el uso de estrategias de afrontamiento activas $y$ en qué momento pasa a potencializar estrategias de afrontamiento pasivas.

Por su parte, la Coopoeratividad sólo correlacionó significativamente con la Expresión Emocional Abierta (EEA), lo cual es diferente a la asociación teóricamente esperada entre Búsqueda de Apoyo Social (BAS) y Cooperatividad (C). Este resultado indica que las personas con alta Cooperatividad tienden a buscar a otras personas, no para generar estrategias para responder a las demandas generadoras de estrés, sino para desahogarse de la tensión emocional. Es interesante este hallaz- 
go según el cual la dimensión del temperamento relacionada con el gregarismo (RD) está asociada con una estrategia activa (BAS) mientras que la dimensión colectivista del carácter (C) lo está con una estrategia emocional (EEA), lo que inicialmente llevaría a suponer que el componente biológico es más favorable para el afrontamiento al estrés mediante la consecución de ayuda de terceros, que el componente social equivalente. No obstante, esto se debe estudiar con mayor detenimiento en futuras investigaciones, pues sus repercusiones pueden ser de gran relevancia académica, en especial en el marco de las teorías evolucionistas de los mecanismos psicológicos adaptativos.

Finalmente, el hecho que la evitación al daño y la persistencia no se relacionaran con ninguna de las dimensiones del afrontamiento estudiadas, se puede deber a un déficit de afrontamiento (paralización) o al hecho de renunciar fácilmente a enfrentar las situaciones generadoras de estrés; en ambos casos los resultados son conceptualmente significativos, pues, por un lado, indicaría que estos jóvenes no valoran adecuadamente los recursos con los que cuentan para afrontar las fuentes de estrés, al punto de paralizarse y esperar pasivamente a que el problema se solucione por sí mismo o que otras personas lo solucionen por ellos; por otro lado, los bajos niveles de persistencia indicarían que esta población renuncia fácilmente a las acciones de afrontamiento, si no reciben retroalimentación positiva e inmediata de las estrategias implementadas para contrarrestar las fuentes generadoras de estrés. Sin embargo, estos aspectos deberán ser estudiados en futuras investigaciones, pues en el caso de persistencia los resultados se pueden deber a una estimación insuficiente del atributo por parte del instrumento empleado.

\section{Referencias}

Arraras, J.I., Wright, S.J., Jusue, G., Tejedor, M. \& Calvo, J.I. (2002). Coping Style, Locus of Control, Psychological Distress And Pain-Related Behaviours in Cancer and Other Diseases. Psychology, Health \& Medicine, 7 (2), 181-187.
Brown, G.K., Nicassio, P.M. \& Woolston, K.A. (1989). Pain Coping Strategies and Depression in Rheumatoid Arthritis. Journal of Consulting and Clinical Psychology, 57, 652-657.

Caballo, V.E. (1998). Manual para el tratamiento cognitivo conductual de los trastornos psicológicos, 2. Madrid, España: Siglo XXI.

Carrobles, J.A., Remor, E. \& Rodríguez A., L. (2003). Afrontamiento, apoyo social percibido y distrés emocional en pacientes con infección por VIH. Psicothema, 15 (3), 420-426.

Cano, E., Quiceno, J., Vinnaccia, S., Gaviria, A., Tobón, S. \& Sandín, B. (2006). Calidad de vida y factores asociados en pacientes con diagnóstico de dispepsia funcional. Universitas Psychologica, 5 (3), 511-520.

Cloninger, C.R. (1994). The Genetic Structure of Personality and Learning: A Phylogenetic Model. Clinical Genetic, 46, 124-137.

Cloninger, C.R., Svrakic, \& Przybeck, T.R. (1993). A Psychobiological Model of Temperament and Character. Arch Gen Psychiatry, 50, 975990.

Cloninger, C.R., Przybeck, T.R.; Svrakic, \& Wetzel, R.D. (1994). The Temperament and Character Inventory (TCl): A Guide to it's Development and Use. St. Louis (Missurri): Center of psychobiology of Personality.

Contreras, F. \& Esguerra, G. (2006). Psicología positiva: una nueva perspectiva en psicología. Revista Diversitas, 2 (2), 311-319.

Contreras, F., Esguerra, G., Espinosa, J. \& Gómez, V. (2007). Estilos de afrontamiento y calidad de vida en pacientes con insuficiencia renal crónica (IRC) en tratamiento de hemodiálisis. Revista Acta Colombiana de Psicología, 10 (2) 169-179.

Díaz, N. \& Pizarro, A. (2000). El modelo psicobiológico de personalidad de Cloninger: Traducción, adaptación y validación del Inventario 
de Temperamento y Carácter en universitarios colombianos. Trabajo de grado no publicado, Universidad Nacional de Colombia, Bogotá, D.C., Colombia.

Espinosa, J.C. \& Herrera, A.N. (2003) Evaluación psicométrica de un instrumento construido por enlace empírico (MMPI). Avances en Medición, 1 (1) 91-104.

Gómez, C., Pérez, M., Portolés, M. \& Salom, R. (2001). Teoría tridimensional de la personalidad: aplicación a los trastornos por abuso de sustancias. Actas Especiales de Psiquiatria, 29 (3), 143-147.

Griswold, G., Evans, S., Spielman, L. \& Fishman, B. (2005). Coping Strategies of HIV Patients with Peripheral Neuropathy. AIDS Care, 17, 711-720.

Gunzerath, L., Connelly, B., Albert, P. \& Knebel, A. (2001). Relationship of Personality Traits and Coping Strategies to Quality of Life in Patients with Alpha-1 Antitrypsin Deficiency. Psychology, Health \& Medicine, 6, 335-341.

Health, A.C., Cloninger, C.R. \& Martin, N.G. (1994). Testing a Model for Genetic Structure of Personality : A Comparison of the Personality Systems of Cloninger and Eysenck. Journal of Personality and Social Psychology, 66 (4), 762 - 775.

Lazarus, R.S. \& Folkman, S. (1986). Estrés y procesos cognitivos. Barcelona, España: Martínez Roca.
Matheny, K.B., Aycock, D.W., Pugh, J.L., Curlette, W.L. \& Silva-Cannella, K.A. (1986). Stress Coping: a Qualitive and Quantitive Synthesis with Implications for Treatment. Counselling Psychologist, 14, 499-549.

Mok, E. \& Tam, B. (2001). Stressors and Coping Methods among Chronic Haemodialysis $\mathrm{Pa}$ tients in Hong Kong. Journal of Clinical Nursing, 10, 503-511.

Richardson, C. \& Poole, H. (2001). Chronic Pain and Coping: A Proposed Role for Nurses and Nursing Models. Journal of Advanced Nursing, 34, 659-667.

Sandín, B. \& Chorot, P. (2003). Cuestionario de afrontamiento al estrés (CAE): desarrollo y validación preliminar. Revista de Psicología y Picopatología Clínica, 8 (1), 39-54.

Van der Zaag-Loonen, H., Grootenhuis, M., Last, B. \& Derkx, H. (2004). Coping Strategies and Quality of Life of Adolescents with Inflammatory Bowel Disease. Quality of Life Research, 13, 1011-1019.

Wahl, A., Hanestad, B., Wiklund, I. \& Poum, T. (1999). Coping and Quality of Life in Patients with Psoriasis. Quality of life Research, 8, 427-433.

Wu, Ch., Lee, Y., Baig, K. \& Wichaikhum, O. (2001). Coping Behaviors of Individuals with Chronic Obstructive Pulmonary Disease. Nursing, 10, 315-320. 
